\title{
Opportunity and Challenge of MOOC on Computer Course Teaching
}

\author{
Weiyu ZHANG \\ School of Information, Qilu University of Technology, Jinan, Shandong, China (zwy@qlu.edu.cn)
}

\begin{abstract}
In recent years, the Massive Open Online Courses (MOOC) increasingly raised people's wide concern. MOOC provides students a new approach to acquire knowledge and a new model to learn. It becomes important learning method in Internet times and brings great impact to college education especially to computer course teaching. In this paper we analyze the emergence and development status of MOOC and discuss the advantages of MOOC. The opportunity and challenge of MOOC on the computer course teaching also is studied. At last we point that we need explore a novel teaching model that more suitable for computer course teaching.
\end{abstract}

Keywords-MOOC, online education, flipped class, reform in education

\section{MOOC 给计算机专业课程教学带来的机遇和挑战}

\author{
张维玉 \\ 齐鲁工业大学信息学院, 济南, 山东, 中国
}

摘 要 近几年, 大规模开放网络课程即 MOOC 越来越受到人们的关注, 它为学习者提供了一种便利的知识获取渠道和学习模式, 成为互联网时代人们学习的新途径, 同时它也将会对现有的高等教育尤其计算机专业课程教学产生巨大的影响。本文深入阐述了 MOOC 的发展现状及分类, 并重点分析了 MOOC 教学模式优势, 深入研究了 MOOC 对计算机专业课程教学带来的挑战和机遇, 以期能够探索出 更适合计算机专业课程教学的新的教学模式。

关键词 MOOC, 在线教育, 翻转课堂, 教学改革

\section{1. 引言}

随着全球互联网技术和教育信息化技术的不断发展, 共享的教育资源和开放的教育形式日益成为教育的热点话 题。其中, 大规模开放在线课程 MOOC (massive open online course）在最近几年迅速发展, 成为一种风靡全球的在线 教学模式。MOOC 的根本目标是让学习者能够方便地利用互 联网络, 根据自己的需求和空闲时间来学习世界上最好的 课程, 从而缓解教育不平衡的现象。

2012 年被称作 “MOOC 之年”, 其标志事件是《纽约时 报》作者帕帕诺发表 “2012 年是 MOOC 之年” 的文章, 从此 MOOC 的发展进入了快车道。目前, 目前国际上最有影 响力的 3 个 MOOC 网站是 Coursera、edX 和 Udacity。 Coursera 是目前规模最大、注册学生最多的在线课程网站, 其学生数已超过 700 万。 edX 是美国麻省理工学院和哈 佛大学联合创办的全球在线教育非盈利组织。而 Udacity 则是在 2012 年 2 月由斯坦福大学教授塞巴斯蒂安 - 斯朗
(Sebastian Thrun) 创建的盈利网站。另外, 2013 年又 被视为中国 $\mathrm{MOOC}$ 元年, 在这一年, 中国多所知名大学纷 纷加入 MOOC 平台。2013 年 5 月, EdX 宣布新增 15 所 高校的在线课程项目, 其中就包括了北京大学、清华大学 两所著名高校。同年 7 月, 上海交通大学宣布加盟 Coursera, 成为加入 Coursera 的第一所中国内地高校。 随后, 复旦大学与 Coursera 达成合作意向, 向 Coursera 免费提供中文或英文教学的在线课程。

MOOC 平台的创建多是起源于美国著名高校, 其课程内 容也最先涵盖计算机专业的多门课程。同时, 计算机专业 课程教学主要以计算机本身作为学习对象, 而 MOOC 的基础 也是一台可以上网的计算机，两者的结合将会产生巨大的 优势, 学生为寻找课程实验的对象而棂费苦心, 而 MOOC 开 放共享的教学模式也将对计算机课程的教学模式带来巨大 的冲击。只需通过一部联网计算机, 教师和全世界的学生 就能进行计算机课程授课、实验、讨论, 而老师甚至可以 
在线监控学生的实验和作业, 在软件的帮助下开展实时评 估。这必将会对未来计算机专业课程教学的改革和发展注 入新的动力。授课教师面对全世界的学习者进行授课, 与 全世界不同文化背景的学生讨论, 自己的思想也会受益匪 浅。因此, MOOC 的发展将为计算机课程教学提出一种新的 思路和方法, 并会对未来计算机课程的学习和教学方法带 来深远而重大的影响。

本文首先介绍了 MOOC 的发展现状及其分类, 然后重点 分析了 MOOC 教学模式的优点, 在此基础上研究了 MOOC 平 台对传统高等教育尤其是计算机专业课程教学带来的机遇 和挑战。最后本文指出我们需要在现有 MOOC 教学方式的基 础上探索新的更适合计算机专业课程教学的教学模式。

\section{2. $\mathrm{MOOC}$ 的现状与三大平台}

MOOC 的本质是通过互联网在全球范围开放在线优质课 程资源, 最大限度地实现名校名师优质课程资源的大规模 学习共享 [1]。MOOC 是在互联网技术成功运用于教育、开 放教育的理念得到社会认可、社会化学习成为一种主要学 习形式的背景下出现的 [2]。该课程模式的出现引起了远程 教育和开放教育乃至整个教育培训行业的广泛关注。按照 学习理论分类, MOOC 可以分为基于关联主义学习理论的 cMOOC ( Connectivist MOOC) 和基于行为主义学习理论的 xMOOC 两种模式 $[3,4]$ 。其中在 cMOOC 课程模式中, 教师 不是课程的主导者, 而是更多地扮演课程发起人和协调人 的角色, 学习者在 $\mathrm{CMOOC}$ 中具有较高的自主性, 学习者在 课前了解课程内容、课中从大量的讨论信息和共享资源中 提取知识、课后又利用包括社交媒体在内的工具继续自发 地交流、组织协作, 构建知识。 XMOOC 则比较接近于传统 教学模式。在学习固定知识点、完成知识的传播和复制方 面, XMOOC 具有很高的效率。不仅如此, xMOOC 学习活动 的设计与展开都针对学习者精心设计, 有利于培养学习者 的兴趣, 有利于保护学习者的求知欲望, 有利于使学习者 掌握获取知识、构建知识的方法。目前最流行的三大平台 Coursera、 edX 和 Udacity 都是基于 xMOOC 模式。

\section{1 Coursera}

Coursera 是目前最大的免费公开在线课程网站, 由美 国斯坦福大学两名计算机科学教授吴恩达 (Andrew Ng) 和 达芙妮・科勒 ( Daphne Koller) 创办, 旨在同世界顶尖大 学合作, 向全球免费提供在线网络课程。Coursera 的首批 合作院校包括斯坦福大学、密歇根大学、普林斯顿大学、 宾夕法尼亚大学 4 所名校。目前, Coursera 平台目前聚 集了来自全球 19 个国家的 108 个教育机构, 包括美国、 欧洲、澳大利亚、中美和亚洲的顶级大学, 为来自世界各
国的 700 万学员提供了超过 600 门免费课程。Coursera 与 高校的合作模式是在双方签订协议达成共识的基础上, Coursera 提供技术开发和支持, 各高校授课教师或团队开 发和设计网络课程, 共同为来自世界各地的学生提供学习 服务和支持。自 2012 年 4 月成立以来, Coursera 的快速 发展令人瞩目, 为全球越来越多的学生提供着高质量的学 习机遇。Coursera 不仅激发了高校及教育工作者利用新 技术改进校园教学的热情, 还在全球范围内掀起了对学习 优质教育资源的渴望, 打破了传统上地域以及成本等带来 的阻碍。

\section{$2.2 \operatorname{edX}$}

edX 是由 麻省理工学院和哈佛大学在 2012 年 5 月 份联合推出的非盈利性教育网站, 旨在以突出的教学设计 为学生提供互动式在线学习。 edX 希望能够继承 MITOCW ( “MIT Open Course Ware”) 开放、共享优质教 育资源的使命, 创建一个反映学科广度和深度, 为学生提 供新的在线学习体验的网络课程平台。开展 edX 项目的目 的不仅是配合校内教学, 提高教学质量和推广在线教育, 而且通过学生学习过程数据的分析, 研究技术在教学中的 应用, 探索混合式教学模式的学习效果。edX 提供的课程 非常丰富主要包括了法律、历史、科学、工程、商科、社 会科学、计算机科学、公共卫生和人工智能等领域。截止 到 2013 年 9 月初, 参与的大学包括: 美国的麻省理工 学院、哈佛大学、加州大学伯克利分校等 28 所世界各地 的顶尖高校。目前, EdX 已经吸引了超过 90 万的学生。 其课程的形式主要由在线视频、网页插入式测试以及协作 论坛组成。 $\mathrm{EdX}$ 平台以交互式学习设计为特色, 平台特征 包括: 自定步调的学习、在线讨论小组、基于 Wiki 的协 作学习、针对学生的学习进程进行及时的评价、在线实验 室和其它的学习交互工具。此平台不仅作为收集和分析学 生学习过程数据的实验室, 而且旨在为学生创设一个世界 范围内的学习共同体。

\section{3 Udacity}

Udacity 是由斯坦福大学教授于 2012 年 2 月创办 的盈利性机构。Udacity 旨在重塑 21 世纪教育, 通过 Udacity 平台给各阶层想学、乐学的人带来可获取的、低 廉的、高参与的高等教育, 来缩小学生技能与就业所需素 质之间的差距。Udacity 已经发布 24 门课程, 分为初级、 中级和高级三个水准, 仅限于商学、计算机科学、数学、 物理学和心理学五个学科领域, 并且着重于实际应用, 比 如教你 “如何建立一个博客”, 以及 “如何构造一个网络浏 览器”。Udacity 的一个目标就是让学生能有更好的职业 
发展。在教师选择上, Udacity 在选择教师时依据的并非 是他们的学术研究能力, 而是他们的教学水平。Udacity 的 课程一部分是由教师自行设计, 一部分是与 Google 或者 微软等公司共同设计推出。Udacity 提供的不仅是单向的 在线视频, 更多的特色在于其高度交互性、基于项目练习 的学, 基于微视频学习的寓教于乐, 基于真实情境的学习, 高度参与的学习社区。

\section{MOOC 的特点}

MOOC 提供的是一种以学习者为中心的在线教学模式, 具有包括课程目标、课程组织者、话题、时间安排、作业 等完整的课程结构。在 MOOC 教学模式中, 课程组织者通 过课程中心平台发布课程内容、组织学习活动、发起讨论 主题, 学习者注册后登录课程中心平台参与到学习中, 并 通过博客、论坛及其他社会性学习工具等记录学习笔记, 分享学习体会, 进行学习交流。课程组织者对学习、讨论 等与学生的互动活动中由学习者生成的内容进行整理和篎 选, 使用电子邮件或者社交媒体软件反馈给学习者。可按 课程的进度学习到最后或者中途放弃, 但完成作业并通过 测试之后, 才可获得该门课程的电子学习证书。与传统的 高校计算机专业课堂教学相比, MOOC 的优势主要体现在以 下几个方面。

\subsection{MOOC 的开放共享全球化思维}

MOOC 之所以成功, 因为它能抛开各种成见与局限, 真 正将资源开放与共享理念运用到实践中, 让资源最大程度 地为大众共享, 为社会服务。这极大改变了资源开放与共 享仅仅停留在小范围内造成优质资源并没有发挥其应有的 作用而造成资料浪费的弊端。因此, MOOC 的一个朴素而又 不新颖的开放共享的全球化思维, 它将全球真正视为一个 地球村, 这样, 它才能超越地域的界限、跨越行业的门槛, 真正为全球具有需求的人士提供帮助; 正是这种开放的、 可共享的、无私的奉献精神往往能打动人, 产生强大的影 响力, 散发出十分迷人的光芒。在 MOOC 现象上, 我们看 到了这种无私的开放与共享的价值是无法衡量, 只有站在 相当的高度, 才能产生相当的影响, 也只有为大家服务, 才能被大家承认和接受。由此可以看出, 在当今世界, 开 放共享的全球化思维是一种基本的而又容易被人忽视的思 维, 开放共享的全球化视野促成了 MOOC 获得巨大的成功。

\subsection{MOOC 具有大规模、多层次的协作交流特点}

MOOC 的视频课程中设置了与学习者的互动环节, 一般 是在完成本节视频单元中知识点的讲解后, 平台以客观题 的形式进行内嵌式的随堂测验, 学习者只有答对问题才能
继续学习。另一方面, MOOC 平台上的课程论坛提供了师生 之间的交流功能。教师可以设置讨论话题, 学生可以就话 题展开讨论, 分享观点, 也可以自主发起话题或提问。其 它学习者可以解答, 也会有专门的课程助理解答。学习者 还可自发利用博客、Facebook、微博、QQ 群等较为熟悉的 平台开发第二课堂, 进行更多形式的互动。

\section{3 MOOC 的重视一线教学思维点}

面对目前我国由于云计算、移动互联及物联网等新兴 信息技术的兴起而诱发的智慧校园的建设热潮, 让我们感 觉到教育信息化似乎又发展到了新到阶段。然而, 目前这 些教育信息化建设工作对教学一线的帮助不明显, 或者说 关注度不够。正因为这样, 才出现了对教学一线的有实质 性帮助的 MOOC, 就立即在全世界范围内掀起了学习 MOOC 的热潮。不管 MOOC 今后发展如何, 它用事实雄辩地证明 了教育信息化的重点应该是在为教学一线服务, 因此, 教 育信息化的出发点与落脚点是为教学一线服务, 只有这样, 教育信息化才能真正找到出路, 才能事半功倍而不是县花 一现。

\section{4 MOOC 全面及时的学习评价和反馈}

除了课程中内嵌式的即时测试之外, MOOC 平台设置还 包括课后测试和平时作业模块。课后测试以客观题为主, 学习者在做答之后即时获得正误反馈和讲解。平时作业一 般是一些开放性的主观题目, 课程组织者公布了详细的评 分标准, 学习者上传作业后由老师或者其他学习者进行评 阅。有的 MOOC 平台甚至规定了每个学习者要评阅规定数 量的他人作业才能算有效完成本次作业。期末考试是学习 者完成本课程学习的最后环节, 学习者只有按 MOOC 平台 的要求参加考试, 成绩合格后才会得到课程学习证书。对 于课程学习过程中所产生的学习内容, 如讨论话题、提交 的平时作业、学习者提出的常见问题等, MOOC 课程组织者 团队会对其进行分析、整理和总结后反馈给学习者。同时, 这些反馈信息又可作为新一轮 MOOC 的课程资源。

\subsection{MOOC 的大数据思维}

MOOC 最重要的价值是创造了一个世界范围内任何人 都可以自由出入的大学堂, 由此推动了工业文明造就的封 闭大学课堂向信息时代开放、共享、多元 “学堂” 的历史 性转变 [5]。MOOC 创造了一个前所未有的网络大课堂, 它 将有用的教学数据视为教师和学生的数据海洋, 它适应了 当今大数据时代的发展要求, 由此客观上符合了以学生为 主体的教育理念的要求, 同时也客观上促进了教师个体向 课程团队的转变以及教师中心向学习者中心的转变。 


\section{MOOC 给计算机专业教学带来的机遇和挑战}

MOOC 打破了大学围墙的界限, 使得学生接受高等教育 不受时间、空间和年龄的诸多限制。目前三大 MOOC 平台 的注册报名人数已超过几百万, 这对社会大众有着非常大 的影响, 对传统的高等教育特别是计算机专业课程教学带 来了极大的挑战。我们相信, MOOC 必将对大学的课程设计 与开发、教学组织、学分认证、师资队伍建设等诸多方面 产生重要而深远的影响, 尤其是教学策略和教学方法方面。 MOOC 的开放特性构成并彰显了其独特的创建、结构以及运 作, 这为传统大学的课程开发、教学组织和运作提供了独 特的思路。

\section{1 以资源共享作为资源建设的重点}

在网络信息时代, 业内人士一致认为高校教育信息化 建设应 “以网络为基础、资源为核心、应用为根本、管理 为灵魂”, 因此资源的核心地位是不容质疑。优质的资源才 是 MOOC 获得广泛认可并迅速推广开来的最根本原因。因此 我们在开展在线教育时, 应该高度重视学校的优质资源, 在视频制作和课程团队建设方面应该给予适当倾斜。另外, MOOC 开放、共享的全球化思维给我们计算机专业课程源的 建设起到很好的启示作用。为了更好地利用本校的优质校 本资源, 我们在资源建设理念上将做一些调整, 即工作重 点由原来资源库建设向资源库管理进行转型, 做到 “少建 库、多修渠”, 侧重做好资源共享的道路建设, 而建库工作 主体向各院系倾斜, 要充分利用各使用单位的人力资源。

\section{2 继续加强信息资源对教学一线的支持作用}

MOOC 成功的一个重要因素就是它对教学一线产生了 实际性作用, 这一点很值得我们思考。因为目前各种新兴 技术的兴起让大家有些应接不暇甚至有些迷失方向, 而 MOOC 的出现无疑给大家指明了一条清晰的道路。因此, MOOC 的出现让我们更加坚定教育信息化工作的重点应该 是支持教学、优化教学过程, 提高教学效果, 只有抓住了 这一根本, 我们的教育信息化事业才不会事倍功半, 才不 会走弯路。我们要想进一步提升计算机专业课程的教学质 量, 就必须进一步加大研究信息技术对教学一线的支持工 作的研究力度, 并稳步推动翻转课堂的建设工作, 研究新 技术对当前教学的支持作用, 做好了一两个成功的案例后 以便在全院乃至全校范围内进行推广。

\section{3 探索大学教学模式创新}

高校教师应转变 “教师在讲台上讲, 学生在讲台下听” 这种传统的传授式教学理念。 MOOC 正是一个改变传统教学
模式的时机，高校应当顺应信息时代发展的趋势和潮流， 积极探索将在线学习、混合学习以及翻转课堂之类的创新 性教学模式有机融合到课堂教学和人才培养中去。在这个 过程当中有许多问题值得我们去研究和探讨。关键在于观 念的改变, 从教师到学生都需要重新审视自己的已有教学 和学习观点。 $\mathrm{MOOC}$ 的平台上, 教师与学生将会充分讨论, 来自全球各地的学习者会将各种问题抛给教师, 也许完全 超乎我们想象。教师的角色也会发生重大改变, 不在于将 知识传授出去, 更多地是在于启发学生以及引导学生进行 更有效的学习。在学生层面, 多年来在固有教学模式下成 长的学生习惯了应试教学模式, 而 MOOC 的教学模式完全 不同。大学可以充分利用现有 MOOC 课程, 鼓励计算机专 业课程教师部分使用或者完全使用现有的高质量的 MOOC 课程。

\section{5. 结束语}

随着在线课程的发展和功能的不断完善, 基于 MOOC 理念构建的高校课程教学模式, 能够突破校园界限, 必将 吸引大量的用户进行在线学习。同时, 随着时间的推进和 学习者数量的不断增加, 在线学习平台中积累了每个学习 者的学习行为数据, 如学习者的选课内容、上网时间和时 长、对某个知识点的学习时长、学习次数、查阅过的学习 资源等数据, 因此可形成教育大数据, 这些教育大数据的 积累又将会为探索新的个性化的教学模式提供重要支撑。 必须指出的是 MOOC 平台的蓬勃发展必将促进高等教育事 业的不断发展, 尤其会对计算机专业课程教学产生深远的 意义。为此, 本文对 $\mathrm{M} 00 \mathrm{C}$ 的现状及其分类做出了深入的讨 论, 并分析了 $\mathrm{MOOC}$ 的优势, 同时提出了 MOOC 对计算机专 业课程教学的机遇和挑战, 最后指出我们应当基于 MOOC 先 进的教学模式积极探索更加适合计算机专业课程教学的新 的教学模式。

\section{参考文献(References)}

[1] Lin Li-jun. The coming of MOOC will change the reform of high education of China. Technology Daily, 2013-10-15 (03). (in Chinese).

[2] Wu Shu-ping. Cloud study environment research under MOOC course model. Software Guide, 2013 (3): 191-193. (in Chinese).

[3] Wang Ping. The latest development and application of massive open online course: From cMOOC to xMOOC. Modern Distance Education Research, 2013(3): 13-19. (in Chinese).

[4] Zhang Zhen-hong, Liu Wen and Han Zhi. The return of learning origin: From OCW to MOOC. Modern Distance Education Research, 2013 (3):20-27. (in Chinese).

[5] Sang Xin-min, Xie Yang-bin and Yang Man-fu. The impact and future of MOOC on college education. High education of China, 2014 (3):3-4. (in Chinese). 